\title{
Research Article \\ Synchronization for a Class of Fractional-Order Hyperchaotic System and Its Application
}

\author{
Wen Tan, ${ }^{1}$ Feng Ling Jiang, ${ }^{1}$ Chuang Xia Huang, ${ }^{2}$ and Lan Zhou ${ }^{1}$ \\ ${ }^{1}$ College of Information and Electrical Engineering, Hunan University of Science and Technology, \\ Xiangtan 411201, China \\ ${ }^{2}$ College of Mathematics and Computing Science, Changsha University of Science and Technology, \\ Changsha 410076, China \\ Correspondence should be addressed to Chuang Xia Huang, cxiahuang@126.com
}

Received 16 January 2012; Accepted 15 April 2012

Academic Editor: Jitao Sun

Copyright (C) 2012 Wen Tan et al. This is an open access article distributed under the Creative Commons Attribution License, which permits unrestricted use, distribution, and reproduction in any medium, provided the original work is properly cited.

\begin{abstract}
A new controller design method is proposed to synchronize the fractional-order hyperchaotic system through the stability theory of fractional calculus; the synchronization between two identical fractional-order Chen hyperchaotic systems is realized by designing only two suitable controllers in the response system. Furthermore, this control scheme can be used in secure communication via the technology of chaotic masking using the complex nonperiodic information as trial message, and the useful information can be recovered at the receiver. Numerical simulations coincide with the theoretical analysis.
\end{abstract}

\section{Introduction}

It is well known that the fractional calculus has a long mathematical history, but its applications to physics and engineering are just recent subject of interest [1]. In recent years, more and more researchers focused on the control of fractional-order chaotic systems and its dynamic behavior [2-4]. In [5], chaos and hyperchaos in the fractional-order Rŏssler equations were studied, in which chaos can exist in the fractional-order Rŏssler equation with order as low as 2.4, and hyperchaos exists in the fractional-order Rosssler hyperchaos equation with order as low as 3.8. In [6], the chaotic behavior with the lowest order 3.72 in the fractional-order hyperchaotic Chen system is presented.

For decades, the complex dynamics and synchronization of chaotic systems have attracted much attention [7-21] since the seminal paper by Pecora and Carroll in 1990 [22]. The chaotic control and synchronization of fractional-order systems are concerned extremely [23-27]. The fractional-order system, compared to the integer-order system, 
has more universality, large key space, and more complex dynamic behaviors than the low-dimensional chaotic system [28], turning into a more challenging work to solve the problem of its control, synchronization, and antisynchronization. In [29], the synchronization of fractional-order hyperchaotic Lorenz system with unknown parameters is realized via designing adaptive tracking controller. In [30], the antisynchronization of different hyperchaotic systems is studied based on the method of active control. In nature, the usual way to synchronize nonlinear fractional-order hyperchaotic systems is to design controller generally needing three or more based on the stability theory of fractional calculus [9, 29-32]. Therefore, such designed controller must result in unnecessarily complicated structure, to a certain extent, which limits to the application in practice.

More recently, studies have been intensively focused on fractional-order hyperchaotic system due to its potential applications in secure communication and control processing. However, to our best knowledge, in literature [33-35], the authors are all concerned with its applications to secure communication with regular or periodic signals. Yet, the normal signals are too simple, and they are easy to be decoded even mingling the chaotic signals with transmission channel. Usually, the actual signal transmission is irregular; therefore, research on the synchronization of fractional-order hyperchaotic systems holds great significance for its application to secure communication with nonperiodic information signals.

In this paper, we propose a new control design method using only two controllers in response system to synchronize a class of fractional-order hyperchaotic system. The example, the two identical fractional-order hyperchaotic Chen systems, is utilized to illustrate how to design the controllers to simplify the existing control scheme, and the proposed procedure can be applied to secure communication via the technology of chaotic masking.

\section{Fractional Derivative and Its Approximation}

There are several definitions of fractional derivatives [1]. In our work, we use the best known Caputo derivative [30] defined by

$$
D_{*}^{\alpha} x(t)=J^{n-\alpha} x^{(n)}(t), \quad \alpha>0,
$$

where $n$ is the first integer which is not less than $\alpha$, and $J^{\beta}$ is the $\beta$-order Riemann-Liouville integer operator which is described as follows:

$$
J^{\beta} y(t)=\frac{1}{\Gamma(\beta)} \int_{0}^{t} \frac{y(\tau)}{(t-\tau)^{1-\beta}} d \tau
$$

where $\Gamma(\cdot)$ is the gamma function and $0<\beta \leqslant 1$.

Given a fractional-order chaotic system, the drive (master) system is

$$
\frac{d^{q} X_{1}}{d t^{q}}=F\left(X_{1}\right)
$$


Here $X_{1} \in \Re^{n \times 1}, F\left(X_{1}\right)=\left(f\left(X_{1}\right), f\left(X_{2}\right), \ldots, f\left(X_{n}\right)\right)^{T}$, and $q \in(0,1]$. The fractional-order $q \in \mathfrak{R}^{n \times 1}$ may be unequal. The equilibrium points of system (2.3) can be derived by solving following equation:

$$
F\left(X_{1}\right)=0 .
$$

The stability of fractional-order system has been thoroughly investigated, and necessary and sufficient conditions have been presented in [26]:

$$
\frac{d^{q} X_{2}}{d t^{q}}=F\left(X_{2}\right)+u(t)
$$

where $X_{2} \in \mathfrak{R}^{n \times 1}$, and $u(t)$ is the control functions. Suppose that the error between the system (2.3) and the system (2.5) is $e(t)=X_{1}(t)-X_{2}(t)$; then the fractional error system can be obtained as

$$
\frac{d^{q} e}{d t^{q}}=F\left(X_{1}\right)-F\left(X_{2}\right)-u(t)
$$

The master-slave synchronization of two chaotic systems is tightly associated with stability of the error dynamics; in this paper, the control function method is presented. Before discussing the method, we first give some useful preliminaries which are of great help to the proof of the forthcoming theorem.

Lemma 2.1 (see [36]). System (2.3) is locally asymptotically stable if all the eigenvalues $\left(\lambda_{1}, \ldots, \lambda_{n}\right)$ of the Jacobian matrix of all equilibrium point satisfy

$$
|\arg (\lambda)|>q>\frac{2}{\pi}
$$

In nature, if $q>(2 / \pi)|\arctan (\operatorname{Im}(\lambda) / \operatorname{Re}(\lambda))|$, the system (2.3) is locally asymptotically stable and behave chaotic for all the variations where the eigenvalues of the matrix satisfy $|\arg (\lambda)|>q \pi / 2$. The stable and unstable regions for $q \in(0,1]$ are depicted in Figure 1. Obviously, the stable region of a fractional-order system is normally larger than its corresponding integer-order system:

$$
\dot{X}_{1}=F\left(X_{1}\right),
$$

whose stable region is the left half plane. Based on this, we obtain the following corollary immediately.

Corollary 2.2. The fractional-order system (2.3) with order $q \in(0,1]$ is asymptotically stable if the corresponding integer system (2.8) is stable. 


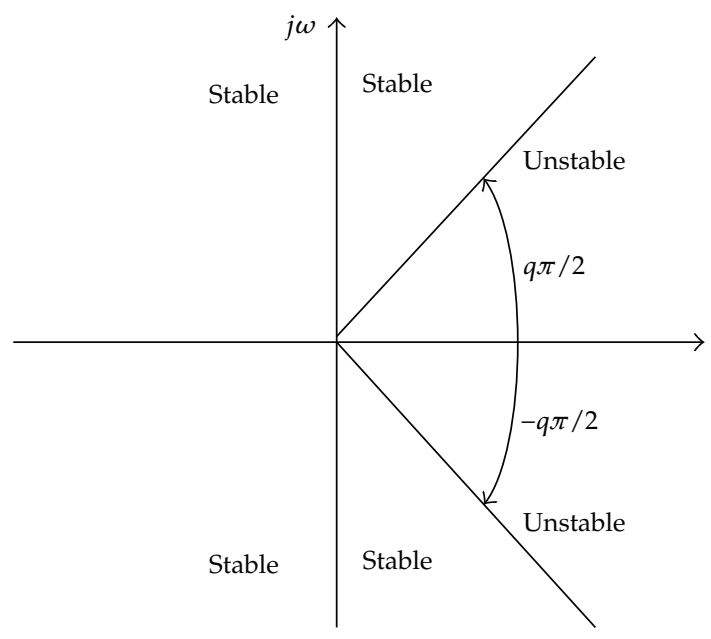

Figure 1: Stability region of fractional-order system.

\section{The Fractional-Order Chen System}

Now, consider the fractional-order hyperchaotic Chen system as follows:

$$
\begin{gathered}
\frac{d^{\alpha} x}{d t^{\alpha}}=a(y-x)+w, \\
\frac{d^{\alpha} y}{d t^{\alpha}}=b x-x z+c y, \\
\frac{d^{\alpha} z}{d t^{\alpha}}=x y-d z, \\
\frac{d^{\alpha} w}{d t^{\alpha}}=y z+r w,
\end{gathered}
$$

where $a=35, b=7, c=12, d=3$, and $r=0.5$. This system has five equilibria:

$$
\begin{aligned}
& S_{0}=(0,0,0,0), \\
& S_{1}=(-82.0531-284.8306 i,-6.6181-1.2245 i,-4.2737+6.9135 i, 12.2500-13.5069 i), \\
& S_{2}=(-82.0531+284.8306 i,-6.6181+1.2245 i,-4.2737-6.9135 i, 12.2500+13.5069 i), \\
& S_{3}=(82.0531-284.8306 i, 6.6181-1.2245 i, 4.2737+6.9135 i, 12.2500+13.5069 i), \\
& S_{4}=(82.0531+284.8306 i, 6.6181+1.2245 i, 4.2737-6.9135 i, 12.2500-13.5069 i) .
\end{aligned}
$$

The corresponding Jacobian matrix is as follows:

$$
J=\left(\begin{array}{cccc}
-35 & 35 & 0 & 1 \\
7 & 12 & -x & 0 \\
y & x & -3 & 0 \\
z & 0 & x & r
\end{array}\right)
$$




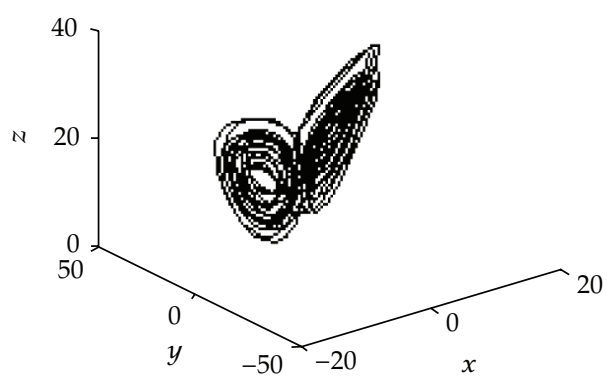

(a)

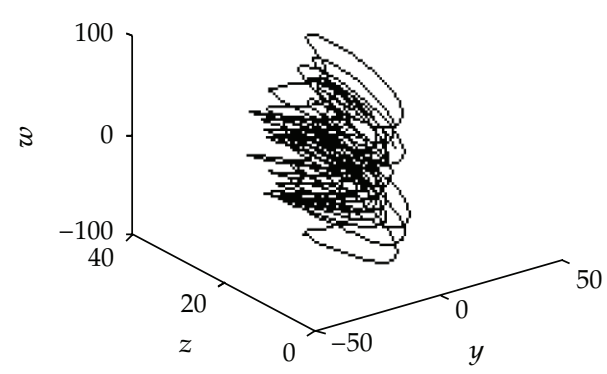

(b)

Figure 2: The phase portrait of system (3.1) chaotic attractor $(\alpha=0.96)$ with (a) $x, y, z$ and (b) $y, z, w$.

Then, the eigenvalues of the Jacobian matrix are obtained:

$$
\begin{array}{llll}
S_{0}: \lambda_{1}=-39.7356, \quad \lambda_{2}=16.7356, \quad \lambda_{3}=-3.0000, & \lambda_{4}=0.5000 ; \\
S_{1}: \lambda_{1}=289.93-82.25 i, & \lambda_{2}=-280.64+81.49 i, & \lambda_{3}=-35.29+0.77 i, & \lambda_{4}=0.50-0.01 i ; \\
S_{2}: \lambda_{1}=289.93+82.25 i, & \lambda_{2}=-280.64-81.49 i, & \lambda_{3}=-35.29-0.77 i, & \lambda_{4}=0.50+0.01 i ; \\
S_{3}: \lambda_{1}=289.93+82.25 i, & \lambda_{2}=-280.64-81.49 i, & \lambda_{3}=-35.29-0.77 i, & \lambda_{4}=0.50 ; \\
S_{4}: \lambda_{1}=289.93-82.25 i, & \lambda_{2}=-280.64+81.49 i, & \lambda_{3}=-35.29+0.77 i, & \lambda_{4}=0.50 .
\end{array}
$$

It is easy to show that eigenvalues from $S_{0}$ to $S_{4}$ hold if they satisfy $q>$ $(2 / \pi)|\arctan (82.25 / 289.93)|$ based on Lemma 2.1, and the fractional-order hyperchaotic Chen is chaotic. From the phase portrait of chaotic attractor at $\alpha=0.96$ as shown in Figure 2 we can find that the system (3.1) exists with chaotic behavior indeed.

\section{The Synchronization of the Two Identical Incommensurate Fractional-Order Chen Systems}

Now, we will study synchronization between two identical fractional-order hyperchaotic Chen systems. The fractional-order hyperchaotic Chen system as the drive system is expressed by

$$
\begin{gathered}
\frac{d^{\alpha} x_{1}}{d t^{\alpha}}=a\left(y_{1}-x_{1}\right)+w_{1}, \\
\frac{d^{\alpha} y_{1}}{d t^{\alpha}}=b x_{1}-x_{1} z_{1}+c y_{1}, \\
\frac{d^{\alpha} z_{1}}{d t^{\alpha}}=x_{1} y_{1}-d z_{1}, \\
\frac{d^{\alpha} w_{1}}{d t^{\alpha}}=y_{1} z_{1}+r w_{1} .
\end{gathered}
$$


And the corresponding response system is written by

$$
\begin{gathered}
\frac{d^{\alpha} x_{2}}{d t^{\alpha}}=a\left(y_{2}-x_{2}\right)+w_{2}, \\
\frac{d^{\alpha} y_{2}}{d t^{\alpha}}=b x_{2}-x_{2} z_{2}+c y_{2}+u_{1} \\
\frac{d^{\alpha} z_{2}}{d t^{\alpha}}=x_{2} y_{2}-d z_{2} \\
\frac{d^{\alpha} w_{2}}{d t^{\alpha}}=y_{2} z_{2}+r w_{2}+u_{2} .
\end{gathered}
$$

Here, $u=\left[u_{1}, u_{2}\right]^{T}$ is the control function. Our aim is to design the controller $u=$ $\left[u_{1}, u_{2}\right]^{T}$ that will make the system (4.2) achieve synchronization with the system (4.1). In order to facilitate the following analysis, we set the errors between the system (4.2) and system (4.1):

$$
\begin{aligned}
& e_{1}=x_{2}-x_{1}, \\
& e_{2}=y_{2}-y_{1}, \\
& e_{3}=z_{2}-z_{1} \\
& e_{4}=w_{2}-w_{1} .
\end{aligned}
$$

From (4.3), (4.2), and (4.1), we obtain the following error dynamical system:

$$
\begin{gathered}
\frac{d^{\alpha} e_{1}}{d t^{\alpha}}=a\left(e_{2}-e_{1}\right)+e_{4}, \\
\frac{d^{\alpha} e_{2}}{d t^{\alpha}}=\left(b-z_{1}\right) e_{1}+c e_{2}+x_{2}\left(z_{1}-z_{2}\right)+u_{1}, \\
\frac{d^{\alpha} e_{3}}{d t^{\alpha}}=-d e_{3}+x_{1} e_{2}+\left(y_{1}+e_{2}\right) e_{1}, \\
\frac{d^{\alpha} e_{4}}{d t^{\alpha}}=r e_{4}+z_{1} e_{2}-y_{2}\left(z_{1}-z_{2}\right)+u_{2} .
\end{gathered}
$$

Then, consider the following control function:

$$
\begin{gathered}
u_{1}=\left(c+k_{1}\right) e_{2}+\left(b-z_{1}\right) e_{1}+x_{2}\left(z_{1}-z_{2}\right), \\
u_{2}=\left(r+k_{2}\right) e_{4}-y_{2}\left(z_{1}-z_{2}\right) .
\end{gathered}
$$


Substituting controllers (4.5) into (4.4), we have

$$
\begin{gathered}
\frac{d^{\alpha} e_{1}}{d t^{\alpha}}=a\left(e_{2}-e_{1}\right)+e_{4}, \\
\frac{d^{\alpha} e_{2}}{d t^{\alpha}}=-k_{1} e_{2}, \\
\frac{d^{\alpha} e_{3}}{d t^{\alpha}}=-d e_{3}+x_{1} e_{2}+\left(y_{1}+e_{2}\right) e_{1}, \\
\frac{d^{\alpha} e_{4}}{d t^{\alpha}}=-k_{2} e_{4}+z_{1} e_{2} .
\end{gathered}
$$

And its corresponding integer system is

$$
\begin{gathered}
\frac{d e_{1}}{d t}=a\left(e_{2}-e_{1}\right)+e_{4}, \\
\frac{d e_{2}}{d t}=-k_{1} e_{2}, \\
\frac{d e_{3}}{d t}=-d e_{3}+x_{1} e_{2}+\left(y_{1}+e_{2}\right) e_{1}, \\
\frac{d e_{4}}{d t}=-k_{2} e_{4}+z_{1} e_{2} .
\end{gathered}
$$
follows:

We can obtain the Jacobian matrix of the error system with linear system (4.7) as

$$
A=\left(\begin{array}{cccc}
-a & a & 0 & 1 \\
0 & -k_{1} & 0 & 0 \\
y_{1}+e_{2} & x_{1}+e_{1} & -d & 0 \\
0 & z_{1} & 0 & -k_{2}
\end{array}\right) .
$$

Obviously, the system (4.7), in which all eigenvalues $-a,-k_{1},-d$, and $-k_{2}$ are less than zero with $k_{1}$ and $k_{2}$ to positive constant, must be stable without a doubt. And the corresponding fractional-order system (4.6) is asymptotically stable according to Corollary 2.2 given in the second section. So, the errors lime $1(t)$, lime $_{2}(t)$, lime $_{3}(t)$, and lime $_{4}(t)$ will converge to zero when $t \rightarrow \infty$. Therefore, synchronization of the two identical fractional-order hyperchaotic systems is achieved.

\subsection{Simulation Results}

In the numerical simulations, we set the parameters of the system (4.1) and (4.2) as $a=35$, $b=3, c=28$, and $r=0.5$ for drive system and response system with $\alpha=0.96$ and the coefficient of control function $k_{1}=15$ and $k_{2}=20$. The initial conditions of the drive and response systems are taken arbitrarily as $x_{1}(0)=10, y_{1}(0)=10, z_{1}(0)=10$, and $w_{1}(0)=10$; and $x_{2}(0)=-10, y_{2}(0)=-10, z_{2}(0)=3$, and $w_{2}(0)=-10$. Numerical results show that the synchronization of two identical fractional-order hyperchaotic system is achieved as shown in Figure 3. The experiments coincided with the theory analysis. 


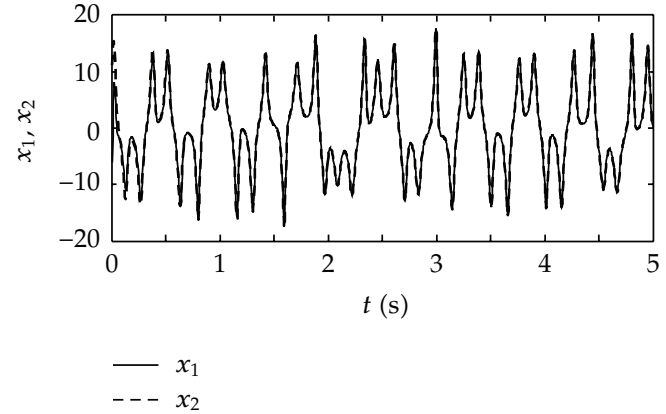

(a)

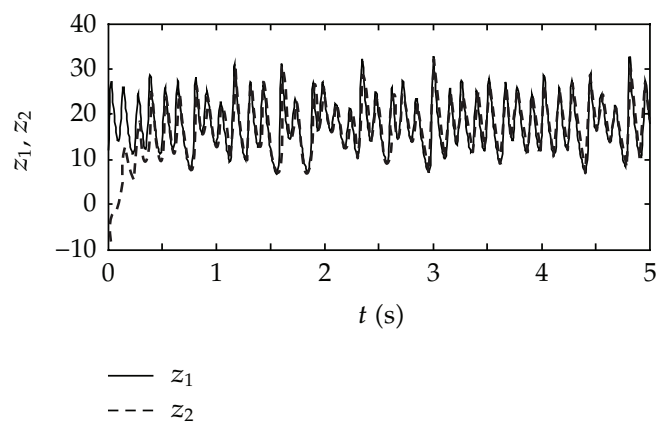

(c)

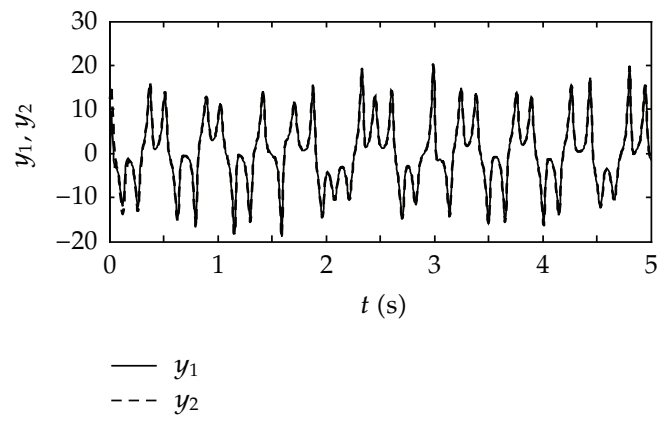

(b)

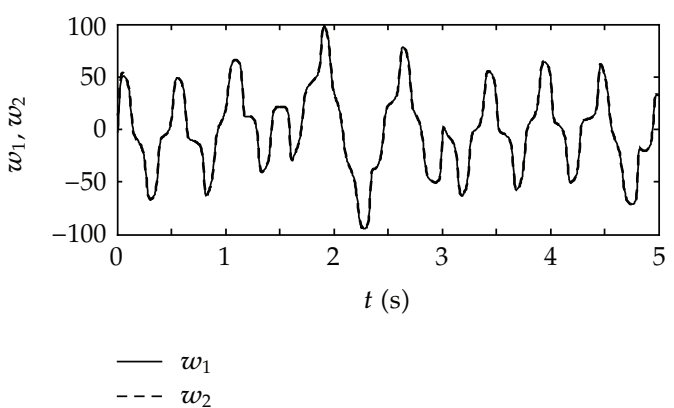

(d)

Figure 3: The synchronization of system (4.2) and system (4.1) with $\alpha=0.96$ : (a) the synchronization of $x_{1}-x_{2}$; (b) the synchronization of $y_{1}-y_{2}$; (c) the synchronization of $z_{1}-z_{2}$; (d) the synchronization of $w_{1}-w_{2}$.

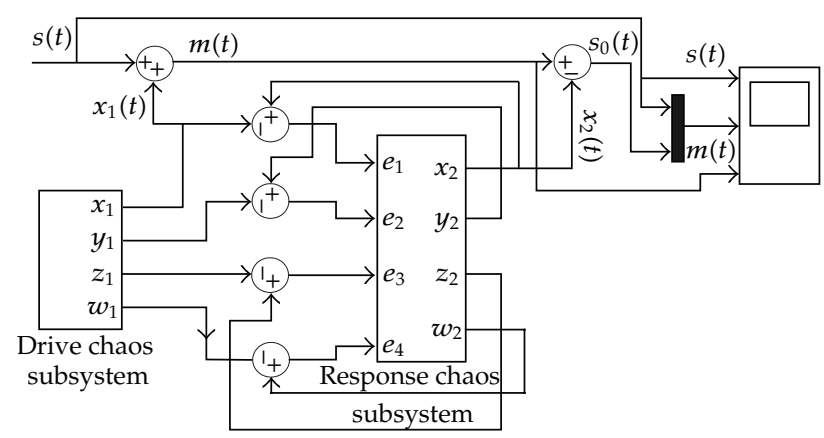

Figure 4: Chaotic masking technology for communication system.

\section{Application to Secure Communication}

In this section, to verify and demonstrate the effectiveness of the proposed method, we will display the numerical results for fractional-order hyperchaotic Chen systems in secure communication. Based on the theory of the communication, the block schematic of secure communication scheme with the synchronization scheme is depicted in Figure 4, where $x_{1}(t)$ is the chaotic state variable of drive system for the transmitter, $x_{2}(t)$ is the chaotic state variable of response system, $s(t)$ is the transmitted message signal with complex nonperiodic 


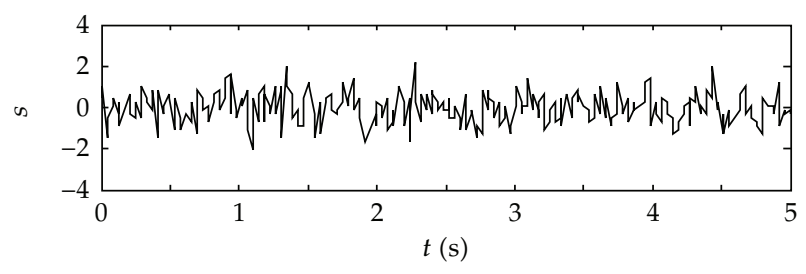

Figure 5: Complex nonperiodic code.

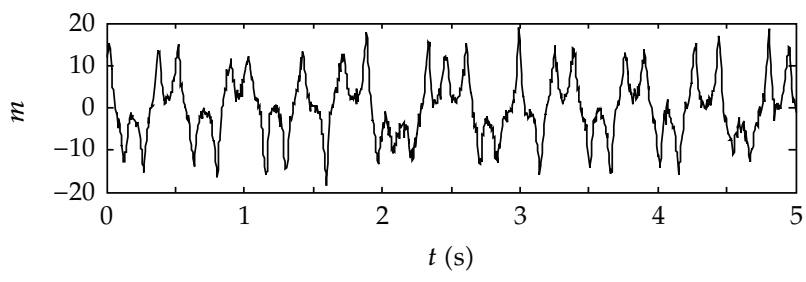

Figure 6: The mixed signals.

mode, which is added to the variable $x_{1}(t)$, mixed signal $m(t)=s(t)+x_{1}(t)$, and $s_{0}(t)$ is the recovered signal after synchronization between the chaotic state variable $x_{2}(t)$ and $x_{1}(t)$ at the receiver terminal end.

The complex nonperiodic information, which has the typical representative to accurately simulate the real transmission signal with generally complex and disorder, is chosen as the transmitted useful message during the numerical experiment in order to reinforce the feasibility of the scheme. The mixed signals (Figure 6) have a good masking effectiveness, are completely different from the original signals (Figure 5), which reached the purpose of safety, and are not to be cracked on the processing of signal transmit. Simulation results show that the system effectively restored the useful signals after about $1.2 \mathrm{~s}$ as depicted in Figure 7. Therefore, we can find that, even adopting other irregular signals as transmitted information by the synchronization and cover-up technology, the useful signals can be recovered with no distortion at the receiver end; namely, the decoded information $s_{0}(t)$ coincides with the transmitted signal $s(t)$.

\section{Conclusions}

In this paper, a new method of designing controller to synchronize a class of fractionalorder hyperchaotic system is presented, and the synchronization between two identical fractional-order systems has been realized via designing only two controllers. The simulation results show that the control method is reliable. Moreover, the complex nonperiodic information signals can be recovered with no distortion when the scheme is applied to secure communication. Numerical experiments for the secure communication system indicate that the synchronization works quite well, which may has potential applications in many interdisciplinary fields. Future work on this topic should include transmission of highfrequency digital signal as well as in-depth studies on application to secure communication. 


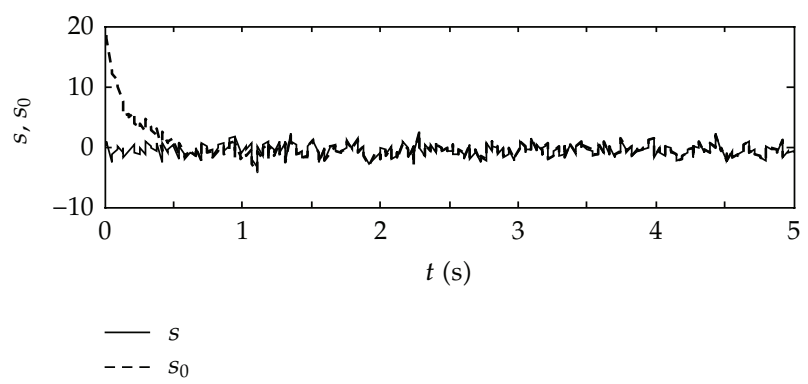

Figure 7: The original message and the recovered message.

\section{Acknowledgments}

The authors are extremely grateful to two anonymous reviewers and particularly to Professor Jitao Sun for their valuable comments and suggestions, which have contributed a lot to the improved presentation of this paper. This work was supported by the Natural Science Foundation of China under Grant nos. 60835004/1101053, the Natural Science Foundation of Hunan Province of China under Grant no. 09JJ3117, the Natural Science Foundation of Jiangsu Province of China under Grant no. BK2009727, the Key Project of Chinese Ministry of Education under Grant no. 211118, the Excellent Youth Foundation of Educational Committee of Hunan Provincial under Grant no. 10B002, and the Planned Science and Technology Project of Hunan Province under Grant no. B11125.

\section{References}

[1] I. Podlubny, Fractional Differential Equations, Academic Press, New York, NY, USA, 1999.

[2] M. S. Tavazoei and M. Haeri, "Chaos control via a simple fractional-order controller," Physics Letters A, vol. 372, no. 6, pp. 798-807, 2008.

[3] P. Zhou, "Chaotic synchronization for a class of fractional-order chaotic systems," Chinese Physics, vol. 16, no. 5, article 16, pp. 1263-1266, 2007.

[4] W. J. Wu, Z. Q. Chen, and Z. Z. Yuan, "Local bifurcation analysis of a four-dimensional hyperchaotic system," Chinese Physics B, vol. 17, no. 7, pp. 2420-2432, 2008.

[5] C. Li and G. Chen, "Chaos and hyperchaos in the fractional-order Rössler equations," Physica A, vol. 341, no. 1-4, pp. 55-61, 2004.

[6] X. Wu and Y. Lu, "Generalized projective synchronization of the fractional-order Chen hyperchaotic system," Nonlinear Dynamics, vol. 57, no. 1-2, pp. 25-35, 2009.

[7] J. Yan and C. Li, "On chaos synchronization of fractional differential equations," Chaos, Solitons and Fractals, vol. 32, no. 2, pp. 725-735, 2007.

[8] Z. Wang, H. Zhang, Y. Li, and N. Sun, "A new method on synchronization of fractional-order chaotic systems," in Proceedings of the Chinese Control and Decision Conference (CCDC '10), pp. 3557-3562, Shenyang, China, May 2010.

[9] R. H. Li, W. Xu, and S. Li, "Linear state feedback control for a new chaotic system," Acta Physica Sinica, vol. 55, no. 2, pp. 598-604, 2006.

[10] W. Tan and Y. N. Wang, "Direct adaptive fuzzy-neural control of unknown chaotic system," Acta Physica Sinica, vol. 53, no. 12, pp. 4087-4091, 2004.

[11] Y. Gu and J. Sun, "Altering synchronizability by adding and deleting edges for scale-free networks," Physica A, vol. 388, no. 15-16, pp. 3261-3267, 2009.

[12] X. Wan and J. Sun, "Adaptive-impulsive synchronization of chaotic systems," Mathematics and Computers in Simulation, vol. 81, no. 8, pp. 1609-1617, 2011.

[13] J. Wang and Y. Zhou, "Analysis of nonlinear fractional control systems in Banach spaces," Nonlinear Analysis. Theory, Methods \& Applications, vol. 74, no. 17, pp. 5929-5942, 2011. 
[14] S. Zhao and J. Sun, "A Lie algebraic condition of stability for hybrid systems and application to hybrid synchronization," International Journal of Bifurcation and Chaos, vol. 19, no. 1, pp. 379-386, 2009.

[15] J. Wang, Y. Zhou, and M. Medved, "On the solvability and optimal controls of fractional integrodifferential evolution systems with infinite delay," Journal of Optimization Theory and Applications, vol. 152, no. 1, pp. 31-50, 2012.

[16] J. Sun, "Some global synchronization criteria for coupled delay-systems via unidirectional linear error feedback approach," Chaos, Solitons and Fractals, vol. 19, no. 4, pp. 789-794, 2004.

[17] H. R. Karimi and H. Gao, "New delay-dependent exponential Hoo synchronization for uncertain neural networks with mixed time delays," IEEE Transactions on Systems, Man, and Cybernetics B, vol. 40, no. 1, pp. 173-185, 2010.

[18] Y. Zhang and J. Sun, "Controlling chaotic Lu systems using impulsive control," Physics Letters A, vol. 342, no. 3, pp. 256-262, 2005.

[19] D. Chen, J. Sun, and Q. Wu, "Impulsive control and its application to Lu's chaotic system," Chaos, Solitons and Fractals, vol. 21, no. 5, pp. 1135-1142, 2004.

[20] F. Wen and X. Yang, "Skewness of return distribution and coefficient of risk premium," Journal of Systems Science \& Complexity, vol. 22, no. 3, pp. 360-371, 2009.

[21] F. Wen and Z. Liu, "A copula-based correlation measure and its application in chinese stock market," International Journal of Information Technology and Decision Making, vol. 8, no. 4, pp. 787-801, 2009.

[22] L. M. Pecora and T. L. Carroll, "Synchronization in chaotic systems," Physical Review Letters, vol. 64, no. 8, pp. 821-824, 1990.

[23] T. D. Ma, H. G. Zhang, and Z. L. Wang, "Impulsive synchronization for unified chaotic systems with channel time-delay and parameter uncertainty," Acta Physica Sinica, vol. 56, no. 7, pp. 3796-3802, 2007.

[24] X. Y. Wang and M. J. Wang, "Dynamic analysis of the fractional-order Liu system and its synchronization," Chaos, vol. 17, no. 3, Article ID 033106, pp. 1-6, 2007.

[25] J. Yan and C. Li, "On chaos synchronization of fractional differential equations," Chaos, Solitons and Fractals, vol. 32, no. 2, pp. 725-735, 2007.

[26] M. S. Tavazoei and M. Haeri, "Synchronization of chaotic fractional-order systems via active sliding mode controller," Physica A, vol. 387, no. 1, pp. 57-70, 2008.

[27] G. H. Erjaee and S. Momani, "Phase synchronization in fractional differential chaotic systems," Physics Letters A, vol. 372, no. 14, pp. 2350-2354, 2008.

[28] H. G. Zhang, Y. Zhao, W. Yu, and D. S. Yang, "A unified approach to fuzzy modelling and robust synchronization of different hyperchaotic systems," Chinese Physics B, vol. 17, no. 11, pp. 4056-4066, 2008.

[29] L. D. Zhao, J. B. Hu, and X. H. Liu, “Adaptive tracking control and synchronization of fractional hyper-chaotic Lorenz system with unknown parameters," Acta Physica Sinica, vol. 59, no. 4, pp. 23052309, 2010.

[30] F. C. Liu, J. Y. Li, and X. F. Zang, "Anti-synchronization of different hyperchaotic systems based on adaptive active control and fractional sliding mode control," Acta Physica Sinica, vol. 60, no. 3, Article ID 030504, 2011.

[31] N. Sun, H. G. Zhang, and Z. L. Wang, "Fractional sliding mode surface controller for projective synchronization of fractional hyperchaotic systems," Acta Physica Sinica, vol. 60, no. 5, Article ID 050511, 2011.

[32] A. Kadir, X.-Y. Wang, and Y.-Z. Zhao, “Adaptive anti-synchronization of unified hyperchaotic system with fully unknown parameters," Acta Physica Sinica, vol. 60, no. 11, Article ID 110510, 2011.

[33] A. Kiani-B, K. Fallahi, N. Pariz, and H. Leung, "A chaotic secure communication scheme using fractional chaotic systems based on an extended fractional Kalman filter," Communications in Nonlinear Science and Numerical Simulation, vol. 14, no. 3, pp. 863-879, 2009.

[34] Y.S. Deng and K. Y. Qin, "Fractional order Liu-system synchronization and its application multimedia security," in Proceedings of the International Conference on Communications, Circuits and Systems (ICCCAS '10), pp. 769-772, Chengdu, China, July 2010.

[35] M. Caputo, "Linear models of dissipation whose Q is almost frequency independent," Geophysical Journal of the Royal Astronomical Society, vol. 13, no. 5, pp. 529-539, 1967.

[36] D. Matignon, "Stability results of fractional differential equations with applications to control processing," in Proceedings of the IMACS-IEEE Multiconference on Computational Engineering in Systems Applications (CESA '96), pp. 963-968, Lille, France, July 1996. 


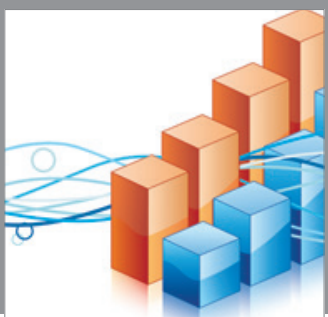

Advances in

Operations Research

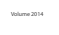

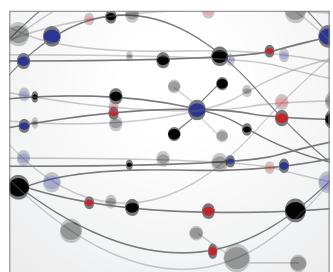

\section{The Scientific} World Journal
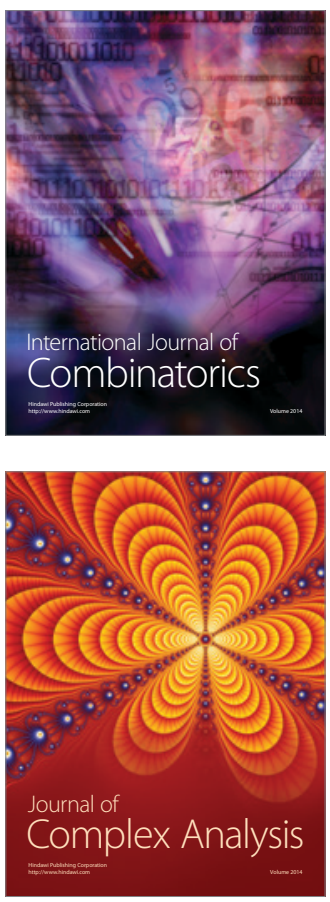

International Journal of

Mathematics and

Mathematical

Sciences
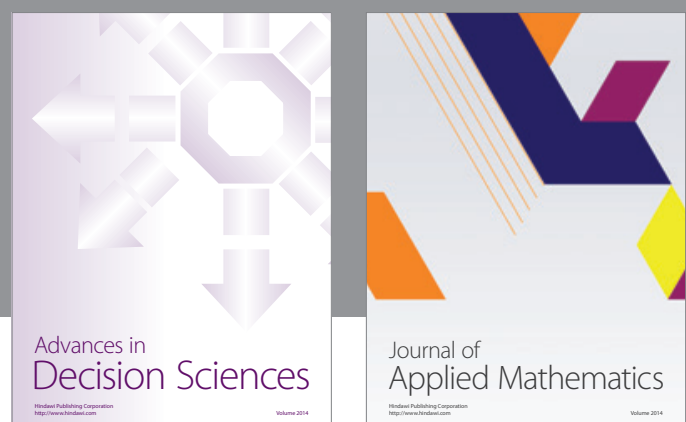

Journal of

Applied Mathematics
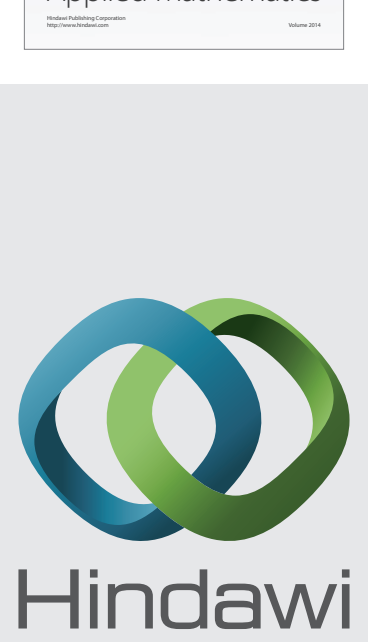

Submit your manuscripts at http://www.hindawi.com
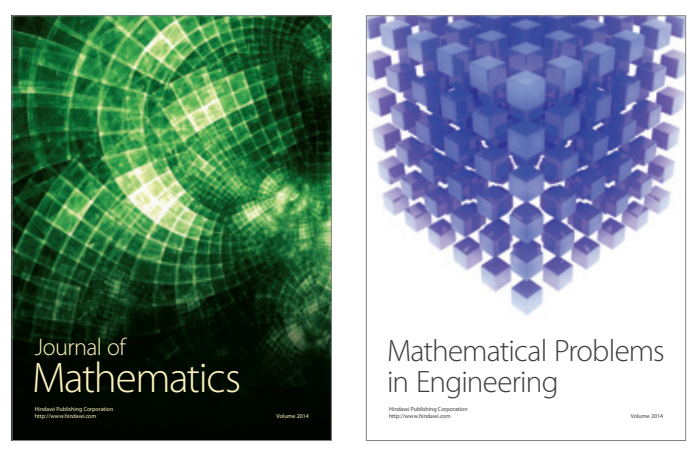

Mathematical Problems in Engineering
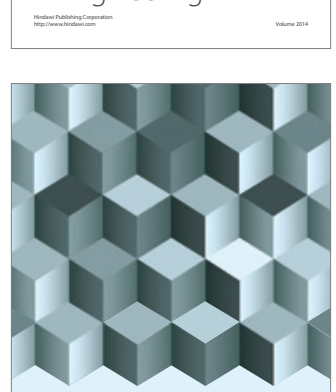

Journal of

Function Spaces
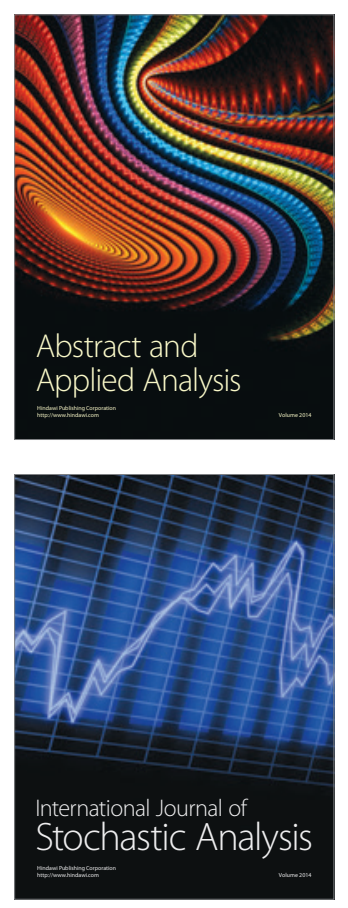

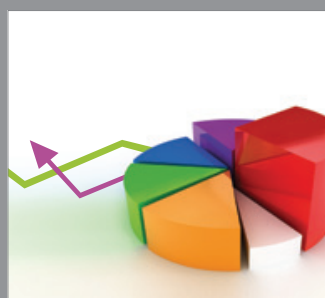

ournal of

Probability and Statistics

Promensencen
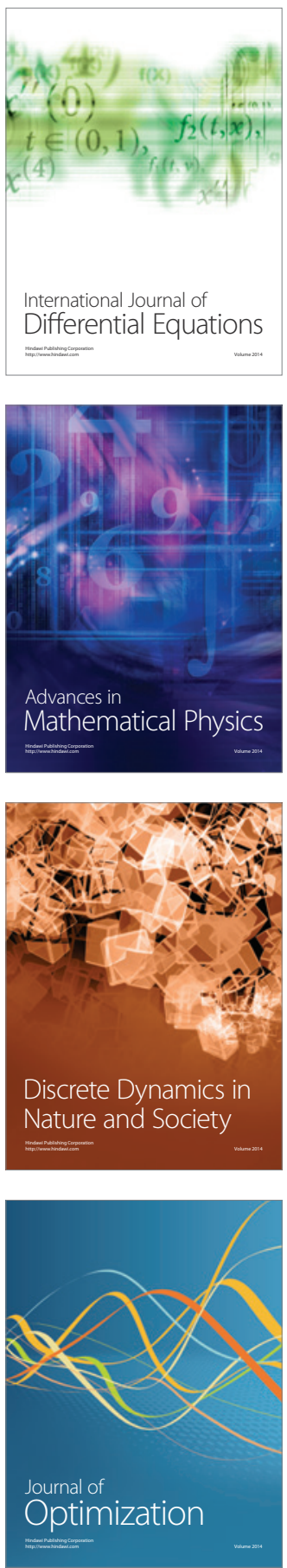